\title{
Cavity QED-based quantum walk
}

\author{
Tiegang Di, ${ }^{1}$ Mark Hillery, ${ }^{2}$ and M. Suhail Zubairy ${ }^{1,3}$ \\ ${ }^{1}$ Department of Physics and Institute for Quantum Studies, Texas A\&M University, College Station, Texas 77843, USA \\ ${ }^{2}$ Department of Physics, Hunter College of CUNY, 695 Park Avenue, New York, New York 10021, USA \\ ${ }^{3}$ Department of Electronics, Quaid-i-Azam University, Islamabad, Pakistan
}

(Received 24 February 2004; published 7 September 2004)

\begin{abstract}
We discuss a possible experimental scheme for the implementation of a quantum walk. The scheme is based on the passage of an atom inside a high- $Q$ cavity. The chirality is characterized by the atomic states and the displacement is characterized by the photon number inside the cavity. The quantum steps are described by appropriate interactions with a sequence of classical and quantized cavity fields.
\end{abstract}

DOI: 10.1103/PhysRevA.70.032304

PACS number(s): 03.67.Lx, 42.50.Pq

It is well known that a quantum computer can solve some problems faster than classical computer. Many classical algorithms used in classical computer are based on random walks. It is therefore interesting to consider the quantum analog of a classical random walk.

There are a number of different types of quantum walk. Discrete-time quantum walks were proposed by Aharonov et al. [1] and further developed by Watrous [2]. These walks rely on an auxiliary quantum system, a "quantum coin," in order to make the time steps in the walk correspond to the action of a unitary operator. Aharonov et al. [3] and Ambainis et al. [4] gave the first explicitly algorithmic context for coined quantum walks. Nayak et al. [5] studied in detail the properties of a quantum walk in one dimension. Farhi and Gutmann [6] introduced continuous-time quantum walks in 1997. Recently Hillery et al. [7] developed a discrete-time quantum-walk model which is based on an analogy to optical interferometers, and does not require a quantum coin. Quantum walks in systems with one and two absorbing walls have also been studied [8].

A number of algorithmic aplications of quantum walks have been found. Childs et al. [9] proved that a continuoustime quantum walk can find its way across a special type of graph exponentially faster than can any classical algorithm. Shenvi et al. [10] demonstrated that a search algorithm based on a coined quantum walk can obtain the same quadratic speedup as Grover's search algorithm. Quantum algorithms that are faster than any classical one have been found for searching databases laid out in $D$ dimensions using a continuous-time walk [11] and in two dimensions using a discrete-time walk [12]. Quantum-walk algorithms have also been found for element distinctness [13], finding triangles in graphs [14], subset finding [15], and determining whether a set of marked elements, which is promised to be of a certain size, exists or not [16].

Methods for the implementation of the coined quantum walk on a number of different physical systems have recently been suggested. These include ion traps [17], neutral atoms trapped in an optical lattice [18], and cavity QED, in which it is the phase of the field that undergoes the walk [19]. Very recently, additional optical implementations have been proposed using either linear optical elements [20,21] or cavities $[22,23]$. In these cavity implementations, the walk takes place among frequency components of the cavity field. These papers also show that an experimental quantum walk has, in fact, been carried out, though it was not interpreted as such at the time [24].

In this paper, we present a study of the properties of quantum walks in one dimension using the cavity QED method. We consider a possible experimental scheme to implement a quantum walk via an interaction between photons and a special two-level atom inside a high- $Q$ cavity. We are interested in a random walk such that the displacement of the particle making the walk corresponds to the number of photons inside the cavity. As photon numbers are always positive, our quantum walk takes place on a straight line with an integer lattice but restricted to a half-space, i.e., it cannot go to negative range. The particle starts at one of those lattice points at some initial time, and at each time step it moves to the left or the right lattice point with equal probability.

A one-dimensional classical random walk can be described as follows. A particle starts at an initial position. The decision to move to the left or right is made by flipping a coin. If the outcome is "heads," the particle moves to the right and if the outcome is "tails," the particle moves to the left. It is well known that the probability of being at a given position remains maximum at the initial position. For a large number of steps, the distribution is given by a Gaussian.

The results for a quantum walk are qualitatively different. The basic difference comes from the fact that, in a quantum walk, we consider the probability amplitudes for the displacement instead of probabilities. As a consequence, there is quantum interference between the probability amplitudes at different locations. One interesting feature is that the probability for location at the initial location is no longer maximum.

In the case of the quantum walk, the particle moves to the left or right according to the outcome of the flip of a "quantum coin" as determined by the chirality [5]. At any point of the lattice the particle has either "left" or "right" chirality. The chirality undergoes a rotation (a unitary transformation called "Hadamard transformation") according to

$$
\begin{aligned}
& |L\rangle \rightarrow \frac{1}{\sqrt{2}}(|L\rangle+|R\rangle), \\
& |R\rangle \rightarrow \frac{1}{\sqrt{2}}(|L\rangle-|R\rangle) .
\end{aligned}
$$

The particle then moves to the adjacent lattice point according to its final chirality state, i.e., 


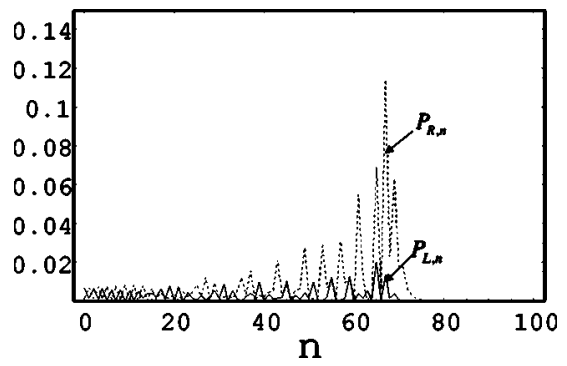

FIG. 1. The probabilities $P_{L, n}$ and $P_{R, n}$ are plotted vs $n$. At the initial time, the particle is located at the position $n_{0}=0$ and the direction of movement is left. Total number of steps is $t=100$.

$$
\begin{gathered}
\left|\psi_{L}(n, t)\right\rangle \rightarrow\left|\psi_{L}(n-1, t)\right\rangle, \\
\left|\psi_{R}(n, t)\right\rangle \rightarrow\left|\psi_{R}(n+1, t)\right\rangle .
\end{gathered}
$$

Here $\left|\psi_{L}(n, t)\right\rangle$ and $\left|\psi_{R}(n, t)\right\rangle$ are the wave functions of the particle at position " $n$ " at time step " $t$ " with "left" or "right" chirality. A simulation of such a quantum walk is presented in Fig. 1. We plot the probabilities $P_{L, n}$ and $P_{R, n}$ which are the probabilities with the left and right chiralities, respectively, for the particle at positions $n$ after $t=100$ steps. The particle is initially located at $n=0$ and moves to the left.

We now introduce a cavity QED scheme for the implementation of the quantum walk discussed above. The proposed scheme is based on the interaction of an atom with an array of classical and quantum radiation fields. However, before describing our scheme, we define certain operations that can be carried out in the atom-field interaction.

(a) First, we consider the resonant interaction of a two-level atom with a classical field. The unitary operator corresponding to this interaction is given by [25]

$$
U_{C}(\theta, \varphi)=\left(\begin{array}{cc}
\cos (\theta) & -i e^{i \varphi} \sin (\theta) \\
-i e^{-i \varphi} \sin (\theta) & \cos (\theta)
\end{array}\right),
$$

where $\theta=\Omega \tau$ with $\Omega$ being the Rabi frequency and $\tau$ the interaction time, and $\varphi$ is the phase of the driving field.

(b) Second, we consider the interaction of a two-level atom with the quantized field inside the cavity and we discuss how a shift of the photon number state can take place via chirping. We assume that the detuning between the atomic transition frequency $\omega_{a b}$ and the cavity resonance frequency $\nu$ is time-dependent (see Fig. 2). The atom-field interaction in the dipole and the rotating-wave approximation is described by the following Hamiltonian:

$\mathcal{H}_{0}=\hbar \nu|a\rangle\left\langle a\left|+\hbar \nu a^{\dagger} a+\hbar \delta(t)\right| a\right\rangle\langle a|+\hbar g\left(|a\rangle\left\langle b\left|a+a^{\dagger}\right| b\right\rangle\langle a|\right)$,

where $\delta(t)=\omega_{a b}-\nu$ is the atom-field detuning. The Hamiltonian can be diagonalized and the atom-field dressed states are given by

$$
\begin{aligned}
& |+\rangle=\cos \theta_{n}|a\rangle|n\rangle-\sin \theta_{n}|b\rangle|n+1\rangle, \\
& |-\rangle=\sin \theta_{n}|a\rangle|n\rangle+\cos \theta_{n}|b\rangle|n+1\rangle .
\end{aligned}
$$

The corresponding energy eigenvalues are

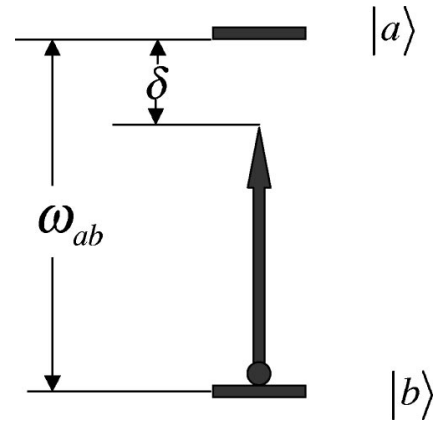

FIG. 2. Schematics of a two-level atom interacting with the radiation field. The energy levels $|a\rangle$ and $|b\rangle$ of the atom are detuned from the radiation field of frequency $\nu$ by an amount $\delta=\omega_{a b}-\nu$.

$$
\begin{gathered}
E_{+n}=\hbar\left[(n+1) \nu+\omega_{a b}\right]-\frac{\hbar}{2}\left[\sqrt{\delta^{2}+4 g^{2}(n+1)}+\delta\right], \\
E_{-n}=\hbar(n \nu)+\frac{\hbar}{2}\left[\sqrt{\delta^{2}+4 g^{2}(n+1)}+\delta\right] .
\end{gathered}
$$

Here

$$
\begin{aligned}
& \sin \theta_{n}=\frac{\sqrt{\delta^{2}+4 g^{2}(n+1)}+\delta}{\sqrt{\left[\sqrt{\delta^{2}+4 g^{2}(n+1)}+\delta\right]^{2}+4 g^{2}(n+1)}}, \\
& \cos \theta_{n}=\frac{2 g \sqrt{n+1}}{\sqrt{\left[\sqrt{\delta^{2}+4 g^{2}(n+1)}+\delta\right]^{2}+4 g^{2}(n+1)}} .
\end{aligned}
$$

We now consider the situation when the atom is initially in state $|b\rangle$ and there are $n$ photons in the cavity. If the atomfield detuning is initially (at $t=t_{i}$ ) such that $\delta=-|\delta|$ with $|\delta|$ $\gg 2 g \sqrt{n+1}$, then we are in a $|+\rangle$ state. Next the detuning is chirped slowly such that, at $t=t_{f}$ (with $\left|t_{i}-t_{f}\right| \gg 2 g \sqrt{n+1}$ ), we have $\delta=+|\delta|$. The atom is then transferred to the $|b\rangle$ with $n+1$ photons. Thus the net result of frequency chirping is that

$$
|a\rangle|n\rangle \rightarrow-|b\rangle|n+1\rangle .
$$

It is not difficult to see that, under the same circumstances, the atom-field state $|b\rangle|n\rangle$ evolves to $|a\rangle|n-1\rangle$. Thus we can describe an operator $S$ such that

$$
\begin{gathered}
S:|a\rangle|n\rangle \rightarrow-|b\rangle|n+1\rangle, \\
S:|b\rangle|n\rangle \rightarrow|a\rangle|n-1\rangle .
\end{gathered}
$$

It may be noted that this transformation takes place regardless of the number of photons $n$ inside the cavity.

Now we are ready to discuss the implementation of a quantum walk based only on the operations $U_{C}(\theta, \varphi)$ and $S$.

We consider the passage of a two-level atom through a cavity. The initial state of the atom can be the ground state $|b\rangle$ or the excited state $|a\rangle$ and the cavity is in the photon number state $\left|n_{0}\right\rangle$. We now show that each step of the quantum walk corresponds to a sequence of the operations $U_{C}(\pi / 2,-\pi / 2) S U_{C}(\pi / 4,-\pi / 2)$. Thus for each step, we need two interactions with the classical fields supplemented 


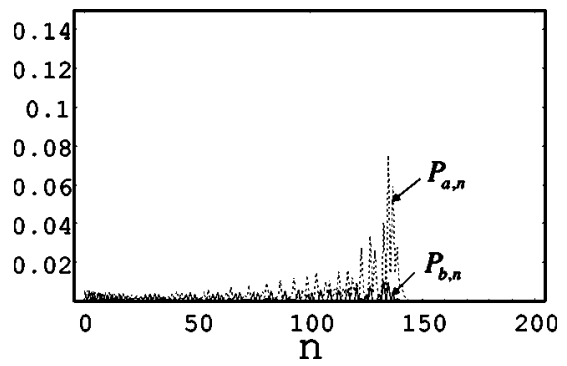

FIG. 3. The probabilities $P_{a, n}$ and $P_{b, n}$ are plotted vs $n$. These plots show the photon numbers after 200 time steps; the initial photon number is $n_{0}=0$.

by a time-dependent interaction with the quantized cavity field.

The atomic states $|b\rangle$ and $|a\rangle$ correspond to "left" and "right" chirality states needed in quantum walks. The photon number states in the cavity represent particle positions. The changing of the photon number corresponds to a particle moving forward or backward. As pointed out earlier, the photon number is non-negative. Therefore, our study concerns only half-space in quantum walks, i.e., the particle is restricted in a non-negative range.

We start the first step of the quantum walk with a Hadamard transformation of chirality states $|a\rangle$ and $|b\rangle$. This step can be simply carried out via interaction between classical field and the two-level atom system. The unitary classical evolution matrix is given as

$$
U_{C}(\pi / 4,-\pi / 2)=\frac{1}{\sqrt{2}}\left(\begin{array}{cc}
1 & -1 \\
1 & 1
\end{array}\right) .
$$

The atomic states $|a\rangle$ and $|b\rangle$ evolve to

$$
\begin{aligned}
& |a\rangle \rightarrow \frac{1}{\sqrt{2}}(|a\rangle+|b\rangle), \\
& |b\rangle \rightarrow \frac{1}{\sqrt{2}}(|b\rangle-|a\rangle) .
\end{aligned}
$$

Please note that there is a slight difference between Eq. (8) and Eq. (1) but it does not affect the final result of quantum walks.

In the second step, we change the photon states according to the following prescription: Photon numbers increase by 1 if the atom is in state $|a\rangle$ and decrease by 1 if the atom is in state $|b\rangle$ without changing the atom states, i.e.,

$$
\begin{aligned}
& |b\rangle|n\rangle \rightarrow|b\rangle|n-1\rangle, \\
& |a\rangle|n\rangle \rightarrow|a\rangle|n+1\rangle .
\end{aligned}
$$

This step cannot be accomplished through a simple Jaynes-Cummings-type interaction. Instead we consider a two-step process. In the first step, we use a frequency chirping method represented by $S$ as discussed in (b) above. The result is $S:|a\rangle|n\rangle \rightarrow-|b\rangle|n+1\rangle$ and $\mathrm{S}:|b\rangle|n\rangle \rightarrow|a\rangle|n-1\rangle$.

Next we use classical evolution [Eq. (3)] with $\theta=\pi / 2$ and $\varphi=-\pi / 2$, i.e.,

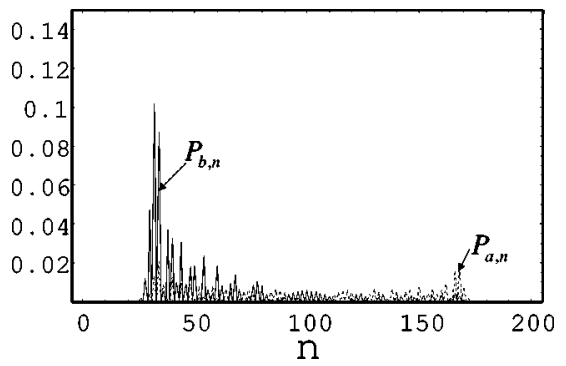

FIG. 4. The probabilities $P_{a, n}$ and $P_{b, n}$ are plotted vs $n$. Here we set the initial photon number $n_{0}=100$, and the number of steps is 100 .

$$
U_{C}(\pi / 2,-\pi / 2)=\left(\begin{array}{cc}
0 & -1 \\
1 & 0
\end{array}\right)
$$

to $\quad$ make $\quad-|b\rangle|n+1\rangle \rightarrow|a\rangle|n+1\rangle$ and

$|a\rangle|n-1\rangle \rightarrow|b\rangle|n-1\rangle$.Thus finally we have

$$
\begin{gathered}
|a\rangle|n\rangle \rightarrow-|b\rangle|n+1\rangle \rightarrow|a\rangle|n+1\rangle, \\
|b\rangle|n\rangle \rightarrow|a\rangle|n-1\rangle \rightarrow|b\rangle|n-1\rangle \quad \text { if } n>0, \\
|b\rangle|0\rangle \rightarrow|b\rangle|0\rangle \rightarrow|a\rangle|0\rangle \quad \text { if } n=0 .
\end{gathered}
$$

The operation of the first and the second steps, i.e., $U_{C}(\pi / 2,-\pi / 2) S U_{C}(\pi / 4,-\pi / 2)$, completes the description of one step of the quantum walk. Repeating these steps again and again we can make quantum walks.

The immediate question arises as to how we can control the classical evolution as well as the time-dependent evolution for chirping during the passage of an atom through the cavity. We propose the atomic levels $|a\rangle$ and $|b\rangle$ to be magnetic sublevels coupled through appropriately polarized light. The interactions can then be controlled via application of a time-dependent magnetic field such that the interaction times for the implementation of the $U_{C}(\theta, \varphi)$ transformation and the time dependence of the detuning $\Delta$ for the chirping are controlled.

In Figs. 3-5, we present results of our simulation based on the solution of the appropriate Schrödinger equation. We choose initial states to be $|b\rangle\left|n_{0}\right\rangle$. For each figure, we give the value of the initial photon numbers $n_{0}$ and the total number of time steps. In these figures, $P_{a, n}$ and $P_{b, n}$ represent the

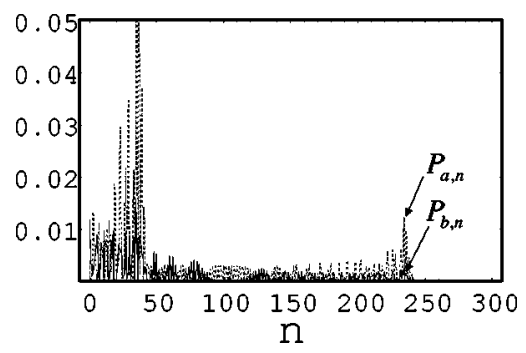

FIG. 5. The probabilities $P_{a, n}$ and $P_{b, n}$ are plotted vs $n$. Here initial photon number is $n_{0}=100$ and total number of time steps is 200. 


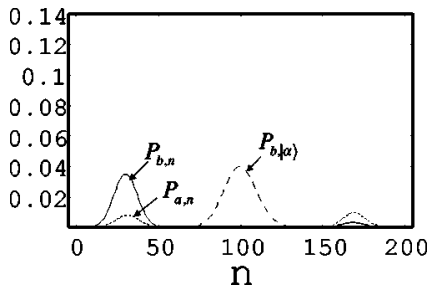

FIG. 6. The probabilities $P_{a n}$ and $P_{b n}$ vs $n$ after time step $t$ $=100$. The initial state of the field is a coherent state $|\alpha\rangle$ and $|\alpha|^{2}$ $=\langle n\rangle=100$. The initial stateof the atom is $|a\rangle$.

probabilities for the atom to be in state $|a\rangle$ and $|b\rangle$, respectively, with $n$ photons inside the cavity. In these simulations, we chose $g /|\delta|=0.01$.

In Fig. 3, we choose $n_{0}=0$ and time step $=200$. The quantum walks in our system cannot go to negative range $(n$ $\geqslant 0$ ). The maximum probability of photon states therefore lies in the range $n=0$ to $n=200$. For a larger number of quantum steps, the maximum probability of photon states moves away from $n=0$ as shown in Fig. 3.

In Fig. 4, we choose $n_{0}=100$ and the number of time steps to be equal to 100 as well. The quantum walks can now take place on both sides of $n=100$. The shape of the probability is not symmetrical and is dependent on the initial atomic state. This is one of the important differences between classical random walk and quantum walk. The maximum probability of photon states lies on the left side of $n=100$ for the initial state $|b\rangle$.

In Fig. 5, we consider the case of $n_{0}=100$ and the number of time steps $t=200$. Here the "particle" moves from $n_{0}$ $=100$ to the right and left as shown in Fig. 4. However, this time the left-going wave will reach $n=0$ and then bounce back to $n>0$. An interference between the left-going and right-going walks leads a complex behavior between $n=0$ and $n=100$. As pointed out earlier, a quantum walk with one barrier has been studied by Bach et al. [8].

At present, it is difficult to have pure photon number states for large photon number. In Fig. 6, we choose initial photon states to be a coherent state $|\alpha\rangle$ and give the final result for a quantum walk. We choose the average photon number to be 100 . The coherent state can be written as a superposition of Fock state and each Fock state undergoes a separate random walk. The final result is obtained by combining all the amplitudes. The net result is an interference of these amplitudes, and the sharp peaks and dips disappear as seen in Fig. 6. The disappearance of the sharp behavior associated with quantum walks will also be expected for any coherent or incoherent mixture of Fock states.

Since classical characters of coherent states are so obvious, it is possible to do experiments to test this kind of coherent state quantum walk.

Decoherence induced by losses through the cavity mirrors $\kappa$ or atomic decay $\gamma$ will also affect the quantum walk as the photon number inside the cavity will change with time. A detailed analysis is complicated and will be presented elsewhere. Here we only point out that the results presented in this paper are valid if the cavity and atomic decay rates are small such that all the steps are completed during a time $t$ $\gg 1 / \kappa, \gamma$.

In conclusion, we have discussed a scheme for the implementation of a quantum walk in a cavity QED system. This system allows us to study the properties of quantum walks in half-space. In the present analysis, we have considered a sequence of classical and quantum interactions of an atom passing through a cavity with a fixed number of photons. Recently, there has been tremendous experimental progress in cavity QED [26,27]. Several aspects of the proposed schemes have been implemented, such as the controlled interaction of an atom with a sequence of classical and quantum field [28]. Fock states with small photon numbers have also been generated [29]. The implementation of the proposed scheme is there within reach at least for small photon numbers and a small number of quantum steps.

The research of M.H. is supported by the National Science Foundation under Grant No. PHY 0139692, and the research of M.S.Z. is supported by Air Force Office of Scientific Research, Air Force Research Laboratories (Rome, New York), DARPA-QuIST, and the TAMU Telecommunication and Informatics Task Force (TITF) initiative.
[1] Y. Aharonov, L. Davidovich, and N. Zagury, Phys. Rev. A 48, 1687 (1993).

[2] J. Watrous, in Proceedings of the 33rd Symposium on the Theory of Computing (ACM Press, New York, 2001), p. 60.

[3] D. Aharanov, A. Ambainis, J. Kempe, and U. Vazirani, in Proceedings of the 33rd Symposium on the Theory of Computing (Ref. [2]), pp. 50-59.

[4] A. Ambainis, E. Bach, A. Nayak, A. Vishwanath, and J. Watrous, in Proceedings of the 33rd Symposium on the Theory of Computing (Ref. [2]), pp. 60-69.

[5] A. Nayak and A. Vishwanath, e-print quant-ph/0010117.

[6] E. Farhi and S. Gutmann, Phys. Rev. A 58, 915 (1998).

[7] M. Hillery, J. Bergou, and E. Feldman, Phys. Rev. A 68, 032314 (2003); E. Feldman and M. Hillery, Phys. Lett. A 324, 277 (2004).
[8] E. Bach, S. Coppersmith, M. P. Goldschen, R. Joynt, and J. Watrous, e-print quant-ph/0207008.

[9] A. M. Childs, R. Cleve, E. Deotto, E. Farhi, S. Gutmann, and D. A. Spielman, in Proceedings of the 35th ACM Symposium on the Theory of Computing (ACM Press, New York, 2003), pp. 59-68.

[10] N. Shenvi, J. Kempe, and K. B. Whaley, Phys. Rev. A 67, 052307 (2003).

[11] A. Childs and J. Goldstone, e-print quant-ph/0306054.

[12] A. Ambainis, J. Kempe, and A. Rivosh, e-print quant-ph/ 0402107.

[13] A. Ambainis, e-print quant-ph/0311001.

[14] F. Magniez, M. Santha, and M. Szegedy, e-print quant-ph/ 0310134.

[15] A. Childs and J. Eisenberg, e-print quant-ph/0311038. 
[16] M. Szegedy, e-print quant-ph/0401053.

[17] B. C. Travaglione and G. J. Milburn, Phys. Rev. A 65, 032310 (2002)

[18] W. Dür, R. Rausendorf, V. M. Kendon, and H.-J. Briegel, Phys. Rev. A 66, 052319 (2002).

[19] B. C. Sanders, S. D. Bartlett, B. Treganna, and P. L. Knight, Phys. Rev. A 67, 042305 (2003).

[20] Z. Zhao, J. Du, H. Li, T. Yang, and J.-W. Pan, e-print quant$\mathrm{ph} / 0212149$.

[21] H. Jeong, M. Paternostro, and M. S. Kim, Phys. Rev. A 69, 012310 (2004).

[22] P. L. Knight, E. Roldan, and J. E. Sipe, Phys. Rev. A 68 , 020301 (2003).

[23] P. L. Knight, E. Roldan, and J. E. Sipe, Opt. Commun. 227, 147 (2003).
[24] D. Bouwmeester, I. Marzoli, G. P. Karman, W. Schleich, and J. P. Woerdman, Phys. Rev. A 61, 013410 (2000).

[25] See, for example, M. O. Scully and M. S. Zubairy, Quantum Optics (Cambridge University Press, London, 1997).

[26] G. Raqithel, C. Wagner, H. Walther, L. M. Narducci, and M. O. Scully, in Advances in Atomic, Molecular, and Optical Physics, edited by P. Berman (Academic, New York, 1994), Suppl. 2, p. 57.

[27] J. M. Raimond, M. Brune, and S. Haroche, Rev. Mod. Phys. 73, 565 (2001).

[28] A. Rauschenbeutel, G. Nogues, S. Osnaghi, P. Bertet, M. Brune, J. M. Raimond, and S. Haroche, Phys. Rev. Lett. 83, 5166 (1999).

[29] S. Brattke, B. T. H. Varcoe, and H. Walther, Phys. Rev. Lett. 86, 3534 (2001). 